Z Herz-Thorax- Gefäßchir 2011 · 25:5-5

DOI 10.1007/s00398-010-0827-5

Online publiziert: 29. Januar 2011

๑) Springer-Verlag 2011

\author{
Klaus Kallenbach \\ Chirurgische Klinik, Klinik für Herzchirurgie, \\ Universitätsklinikum Heidelberg, 69120 Heidelberg
}

\section{Zurück in die Zukunft}

den. Die neu geschaffene Rubrik „Blick über den Tellerrand" soll sich erfrischend von wissenschaftlichen Textmustern abheben und den Einblick in andere Bereiche, die gelegentlich ausgesprochen ungewöhnlich mit der Herzchirurgie verbunden sind, beleuchten. Wir wollen den geneigten Leserinnen und Lesern in jeder Ausgabe unserer Zeitschrift einen bunten Strauß verschiedenartiger Beiträge bieten, wodurch eine entspannte und weniger verpflichtende Form der Informationsvermehrung erreicht werden soll.

Erstmalig haben wir in dieser Ausgabe versucht, ein Diskussionsforum zu schaffen: parallel zum Artikel von Herrn Dr. Börgermann über die OPCAB-Chirurgie baten wir vier gestandene Herzchirurgen, einen Kommentar zum Thema OPCAB zu verfassen, ohne den Beitrag gelesen zu haben. Ungeachtet der Methoden evidenzbasierter Medizin gelingt es nicht immer, ein herzchirurgisches Verfahren eindeutig zu bewerten - die Kommentare sollen helfen, die derzeit stattfindende, zum Teil kontroverse Diskussion besser fassbar zu machen. Dabei hoffen wir außerdem, dass sich unsere Leserschaft langfristig aktiv mit Beiträgen oder Leserbriefen an den angestoßenen Diskussionen beteiligt.

Zusammen mit dem Herausgebergremium und dem Springer-Verlag hoffen wir, Ihnen weiterhin eine informative und unterhaltende Zeitschrift für Herz-, Thorax- und Gefäßchirurgie bieten $\mathrm{zu}$ können, deren Lektüre weiterbildet und Freude bereitet!
Mit herzlichen Grüßen

Ihre
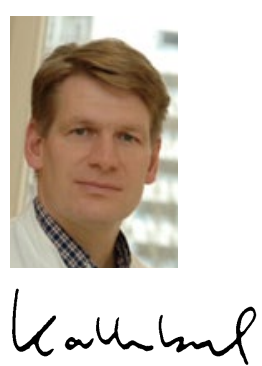

Priv.-Doz. Dr. Klaus Kallenbach
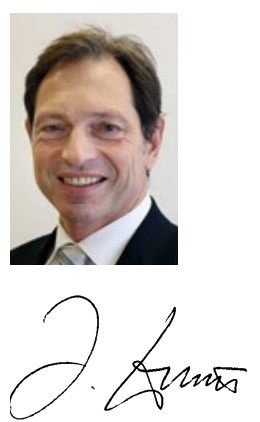

Prof. Dr. Jochen Cremer

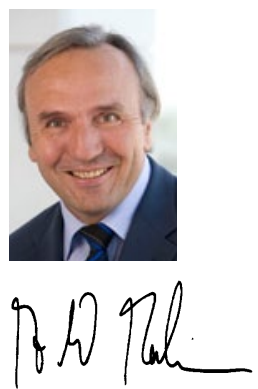

Prof. Dr. Friedrich-Wilhelm Mohr

\section{Korrespondenzadresse \\ PD Dr. Klaus Kallenbach}

Chirurgische Klinik, Klinik für Herzchirurgie, Universitätsklinikum Heidelberg Im Neuenheimer Feld 11069120 Heidelberg

klaus.kallenbach@med.uni-heidelberg.de 